\title{
Pragmatic Functions of Fuzzy Language and Its Translation
}

\author{
Hailiang Ning \\ Taishan University, No.125 Taishan Road, Taian 271012, China \\ E-mail: ninghailiang689@163.com \\ Kun Wang \\ Foreign Languages School, Ludong University \\ 186 Hongqizhonglu Road, Yantai 264025, China \\ E-mail:pjx2001@163.com \\ Fachun Zhang (Corresponding author) \\ Foreign Languages School, Ludong University \\ 186 Hongqizhonglu Road, Yantai 264025, China \\ Tel: 86-535-492-3230 E-mail: timothyzfc95@sina.com
}

$\begin{array}{lrr}\text { Received: June 6, } 2011 & \text { Accepted: October 24, } 2011 & \text { Published: January 1, } 2012 \\ \text { doi:10.5539/ass.v8n1p253 } & \text { URL: http://dx.doi.org/10.5539/ass.v8n1p253 }\end{array}$

\begin{abstract}
In this thesis, the author expounds pragmatic functions of fuzzy language systematically on the basis of the brief introduction of its definition and attributes, and then proposes three strategies for the translation of fuzzy language.
\end{abstract}

Keywords: Fuzziness, Pragmatic functions, Translation

\section{Introduction}

Fuzziness, which means the property of fuzzy language whose boundary is not definite, is attached great importance by experts at home and abroad for a long time. At the very beginning of the thesis, it is significant to have a brief review of the history of the study on fuzzy language. In western countries, Eubulides is the first person to notice fuzzy characteristic of natural language. After several years C. S. Perce takes the lead in formulating the notion in a rigorous way(Channel, 1994: 7). He thinks that it is possible for speakers to produce utterance even they didn't know the exact boundary of them. In 1965, A Zadeh put forward the Fuzzy Set Theory, which is published in Information and Control. Thereby, a host of disciplines sprang up, including fuzzy mathematics, fuzzy psychology, fuzzy logic and fuzzy linguistics.

From then on, great attentions have been drawn from linguists. For instance, American linguist Lakeoff (1973) applies Fuzzy Set Theory to word meaning; American scholar, Prince together with his cooperators find that fuzzy language is universal in our language (Prince \& Bosk, 1980: 83-97). British mathematician Guilbaud suggests fuzziness is indispensable for our communication (Guilbaud, 1997). Australian linguist Burns also achieves a lot in fuzzy language.

In China, Wu Tieping takes the lead in introducing fuzzy language research to domestic linguistic field. Following Wu's footsteps, quite a few linguists begin to focus on language fuzziness. In 1979, Zhang Qiao (1998a) starts semantic fuzziness. Shi Anshi (1988) also publishes an article about fuzzy semantics. Besides the study of fuzzy language from the perspective of semantics, there are still some experts paying attention to pragmatic fuzziness. Wu Tieping (1999) once refers to it in Fuzzy Linguistics. He Ziran (1985) focuses on the pragmatic analysis of hedges. As a matter of fact, fuzzy language has its distinct pragmatic functions and is employed in many fields.

This thesis adopts the method of analyzing the data collected from all sorts of writings and it is composes of five 
parts, namely, introduction, fuzzy language and its attributes, pragmatic functions of fuzzy language, strategies for translation of fuzzy language and conclusion, based on the careful analysis of the pragmatic functions of fuzzy language, strategies for translation of fuzzy language are proposed so as to give some useful suggestion in the process of translation.

\section{Pragmatic Functions of Fuzzy Language}

For the distinctive attributes fuzzy language performs certain functions in our daily life. In this chapter, we would focus on its functions from the perspective of pragmatics, which is the study of language in use, and concerned with practical results and values. That is to say, we would study the functions of fuzzy language in context. The author here demonstrates positive functions, including improving accuracy, improving flexibility, conveying meaning efficiently, conveying meaning politely and appropriately and negative functions.

\subsection{Positive Functions of Fuzzy Language}

\subsubsection{Improving Accuracy}

Wittgenstein (1953) argues that: "Is it even always an advantage to replace an indistinct picture by a sharp one? Isn't the indistinct one often exactly what we need?" People usually think precise language is good for communication while in some cases, the use of fuzzy language can improve the accuracy of expressions. H.P. Grice (1975:45) put forward Cooperative Principle in 1967. He thinks that in order to make communication smooth and successful, we should obey four principles ,namely, the maxim of quality, the maxim of quantity, the maxim of relation, the maxim of manner. Fuzzy language seems to deny the quantity maxim, but in this way it obeys the quality maxim and guarantees the accuracy of information concerned. For instance,

a. She is about six years old.

b. The doctor said there was something wrong with your stomach.

Sentence a. uses the approximator "about" because the speaker is not sure about it. If the speaker says: "she is six years old" the hearer might be misled. "Something" in sentence b. suggests that the stomach is not in a healthy state. The doctor is not sure whether it is serious or not, and the fuzzy word "something" helps avoid false information. British law of slander formulates: "for any term not exceeding two years, and to pay such fine as the court shall award." "Not exceeding two years" only shows the upper limit of time and "as the court shall award" does not explain the exact amount of money. Thus, it can protect the legal right of the people concerned.

The American military command said today that two Iraqi police officers were killed on Friday and more than 350 people wounded or sickened in three suicide attacks in Anbar province using trucks filled with chlorine gas (www.nytimes.com)

In the above news, when referring to the number of wounded people, the author uses fuzzy expression "more than 350 people" News reporters are asked to report the latest news as quickly as possible. Sometimes it is impossible for them to know the exact details in a short time. The use of fuzzy words ensures the accuracy of news information and also convinces the readers.

\subsubsection{Improving Flexibility}

For something we are not sure about or lack sufficient information fuzzy language can not only improve accuracy but also improve the flexibility of expressions. For example, "He may be at home". In this sentence the speaker is not very sure whether he is at home or not. The expression "may be" avoids being too rigid and absolute, provides possibilities of changing ideas, and gets rid of the unnecessary responsibility caused by the uncertain information, thus, protecting ourselves. For this attribute fuzzy language is applied extensively to many fields.

For the market situation is changing constantly, in international business negotiation, businessmen employ fuzzy language for the sake of maximum profits and avoiding unnecessary troubles and disputes. For example,

We shall do our best to make shipment as soon as possible.

Our price compare favorably with those offered by other manufacturers either in Europe or anywhere else, and we are one of the best manufacturers in this line. Here are our latest price sheets; you'll see our prices are most comparative.

In the first example, "do our best" is a kind of wish, and it does not explain what measures they will take. "As soon as possible" is very fuzzy and does not point out the exact time. By using fuzzy language, they avoid being too rigid, gain time for themselves and leave a good impression to their cooperator.

Also, in news it plays an important role. News reports stress speed and news agency would report the latest news 
as quickly as possible. As a result, sometimes the news reported is not exact or even not correct for lacking enough time to make a thorough investigation about it. While fuzzy language, which serves as a shield, provides for news especially for international important news, the possibility to change the data, helping avoid the unnecessary responsibility led by inaccurate data. For instance,

According to the aide, militants launched mortars and fired machine gun rounds at the 10-car convoy of Minister of Higher Education and Scientific Research Abed Dhiyab al-Ajihi, a sunni Arab member of prime minister Nuri al-maliki's Shiite-led government. (cnn)

"We need to know that else was allegedly done to these detainees before immunity could even be considered." the source said.

Fuzzy words "according to" and "the source said" do not say exactly where the information comes from. In case the information is proved to be inaccurate, fuzzy words can transfer the responsibility and free news agency from troubles.

\subsubsection{Conveying Information Efficiently}

With resort to fuzzy language we can not only improve accuracy but also convey information efficiently. That is to say, by using fuzzy language, we can make less effort to convey a large amount of information, and manage or judge complicated matters with high efficiency. Occasionally, precise words seem very wordy and unnecessary while fuzzy language is more appropriate. Take the dialogue between one patient and a doctor as an example.

Doctor: Serious, your headache?

Patient: Yes

Doctor: Fever?

Patient: A little

Doctor: How long does it last?

Patient: Several days

Doctor: Why not come earlier?

Patient: Too busy

The patient uses "a little" "yes" "several days" and "too busy" to answer these questions. Thus, the doctor can have a rough knowledge about the physical conditions of the patient in a very short time. If the patient uses precise words, it would waste a lot of time, and the doctor would become impatient. Besides, advertisements would employ fuzzy language to inform the audience in a conciser and more informative way.

Extra taste, not extra calories.

Let's make things better.

One word "extra" conveys sufficient information about the product. Audience would know the new features of this food. We save time and efforts, and the advertisement impresses the audience deeply, which helps promote the sales. Similarly, the second advertisement extends a large amount of information by employing limited words.

When we describe a person we would say: "he is very tall, a little fat, round -faced, and wears glasses" The information is enough for readers to have some knowledge about the person and form a rough image quickly. Instead, if we say: "he is 1.78 meters high, 80 kilometers, round-faced and wears glasses." We have to do a lot of work in our minds so as to have an impression about him. It is really a waste of time and the efficiency is lowered.

\subsubsection{Conveying Information Politely and Appropriately}

British linguist G.N.Leech (1983:131-144) formulates the Politeness Principle in 1983, that is tact maxim, generosity maxim, approbation maxim, modesty maxim, agreement maxim and sympathy maxim. Fuzzy language and politeness are connected closely. The politer you would be the more fuzzy words you should employ. Especially in some sensitive political issues and business negotiations, the use of fuzzy language can create a relaxed and harmonious atmosphere, maintain esteem and let others feel your sincerity and politeness. Suppose one of your friends buys a T-shirt and asks your opinion about it. In fact you do not think it is very nice but you'd better say like this: "it is OK, but the colour is a little bit darker." No matter whether your friend would accept your suggestion or not, he or she would not be annoyed by your words. 
Fuzzy language is also very essential in business community for it lets the communication go on in a pleasant atmosphere. Let's see the following example.

As for goods Article 80, we are not able to place an order with you because another supplier is offering us the similar quality at a lower price.

From this article we can find that the company would not make an order with this supplier, whose price is too high. Nevertheless, the company does not express his intention directly but uses "another supplier", "simple quality" and "lower price" to implicate his dissatisfaction about the price politely. Thus, the cooperator will preserve esteem and accept the article pleasantly.

\subsection{Negative Functions of Fuzzy Language}

Fuzzy language indeed assumes a crucial role in many fields, but it does not mean we can always use it. Occasionally, it will do harm to our communication for not knowing the exact meaning. What's worse, people might be cheated and deceived by some fuzzy expressions. In our daily life, we can find some advertisements use fuzzy language to mislead people for the sake of making profits. For example,

Mai yi zeng yi

We can often find such slogan in promotion activities of manufacturers. People usually would buy the product just for the "one" gift, but they often find the so-called Mai yi zeng yi turns out to be something like "one bottle of shampoo with one free -of -charge plastic comb", manufacturers use blurring number "one" to confuse consumers for the purpose of increasing sales.

Let's see one military order: "send out before dawn." The word "dawn" is a kind of fuzzy expression and does not tell the exact time of sending out so it is difficult to send out at the right time and finish the task. Here it is inappropriate to use fuzzy language.

From the above we know that fuzzy language has both positive and negative functions. We should pay attention to the both sides of fuzzy language and use it in an appropriate way, making it perform the positive functions and avoiding its negative functions, thus, letting our communication go on smoothly and pleasantly.

\section{Strategies for Translation of Fuzzy Language}

Fuzziness is an innate characteristic of language, which is found across different languages. In many cases, translators are required to render fuzzy expressions into corresponding ones. It is necessary to have some knowledge of strategies for translation of fuzzy language. In this chapter, three strategies are proposed: fuzziness to fuzziness, deletion of fuzziness, and addition of fuzziness.

\subsection{Fuzziness to Fuzziness}

"Fuzziness to fuzziness" is an important strategy for translating fuzzy language, which can be realized in two ways. One is to render literally fuzzy expressions into counterparts. The other is to render the

fuzzy expressions into the cultural substitutions for we can not find the equivalent ones in target language. Words like "red" "orange" "blue" and "yellow" are fuzzy words and we can translate literally them into hong se, cheng se, lan se, he huang se. But we translate "hot spring" into wen quan.Other examples are cited below.

\subsection{Deletion of Fuzziness}

Another method of translating fuzzy language is to delete fuzziness. By this method the translator would omit some information that is considered to be unnecessary, irrelevant and of little importance. Thus, the translation would be clear and easy to be understood. Otherwise, the translation would seem very redundant and confusing. For instance, when we try to translate the sentence ta zhen shi yi ge wu yong you hei zhui de dong xi. we'd better translate it like this: he is an white elephant. Suppose we render it word by word, it will be a troublesome task and readers may feel puzzled. Besides, the following may be served as a good example.

Wei yu shang hai jiu jiang lu de jin rong guang chang, xiong ju huang pu jiang shang ye zhong xin dexin zang di dai, jiao tong fang bian, shang mao wang lai pin fan, di dian shi zhong.

In the heart of Hangpujiang, Financial Square on Jiujiang Road is well-situated shanghai office tower.

In this example, the translator omits jiao tong fang bian, shang mao wang lai pin fan but says directly "a well-situated shanghai office tower". It is an advertisement for promoting land. By omitting some information, businessmen can catch the important information easily. Otherwise, it is not so easy to understand the main purpose of advertising. Sometimes omitting some information is better than preserving them. We should choose the appropriate way according to different contexts. 


\subsection{Addition of Fuzziness}

The third way to translate fuzzy language is to add fuzziness in target language, which means that we would add some information not contained in the original texts when translating. According to the addition of fuzziness, we can make our translation expressive, complete and specific, which helps grasp the meaning of the original text and avoid confusion. But it does not mean we can add information randomly. We should supplement the necessary information according to the meaning of the original texts. Take the advertisement of Sum Sung as an example: taking lead in a digital world. The suggested translation is ling xian shu ma chao yue yong heng.From the Chinese version we can find that chao yue yong heng is added. In this way, the meaning is enriched, showing that it can not only take a leading role in digital world, but also decides to surpass the best. Audience would be attracted by the excellent advertisement. In addition, there are some other examples to illustrate the point.

The high-altitude plane was and still is a remarkable bird.

Gao kong fei ji guo qu shi xian zai reng ran shi yi zhong liao bu qi de fei xing qi.

There is no such words as "in the past" and "now" in the original sentence, but we add guo qu and xian zai when translating. Verbs "was" and "is" do tell us the different time. If we do not add guo qu and xian zai to the translation it might be difficult for target readers to understand it.

To conclude, in the above we have introduced three ways to translate fuzzy language: fuzziness to fuzziness, deletion of fuzziness, addition of fuzziness. The three ways have their own advantages and shortcomings and their fields of application are different. Given to this situation, we should employ them according to contexts, letting our translation acceptable and readable.

\section{Conclusion}

Fuzziness is an inherent characteristic of natural language. Many efforts have been made by experts at home and abroad on this topic and great success has been achieved. In this thesis, the author first explicates the concept of fuzziness: fuzziness is the innate property of language whose boundary is not clear-cut. Then the author proceeds to introduce three attributes of fuzzy language: indeterminacy, determinacy and relativity. Indeterminacy refers to the indefinite boundary of fuzzy language, while determinacy indicates there is a definite core no matter how fuzzy it is. Relativity requires people to interpret fuzzy language according to contexts. Explaining the definition and attributes of fuzzy language paves the way for identifying the pragmatic functions of fuzzy language, which are classified into positive functions, namely, improving accuracy, improving flexibility, conveying information efficiently, conveying information politely and appropriately, and making language aesthetic and negative function. When speaker is not very sure about the information concerned, fuzzy language ensures its accuracy, otherwise, the hearer may be misled. Besides, for something we are not sure of, fuzzy language can also improve its flexibility, avoiding being too rigid. We can change the information which is proved false and get rid of the trouble caused by the uncertain information. Fuzzy language also performs the function of conveying information efficiently, which means we can make less effort to convey a large amount of information. Next function is conveying meaning politely and appropriately, which is employed in some sensitive political issues. In addition, fuzzy language makes our expressions more vivid and impressive. However, fuzzy language has negative functions. It can cause misunderstanding or cheating activities if used inappropriately. Last but not least, we discuss the strategies for translation of fuzzy language: fuzziness to fuzziness, deletion of fuzziness, and addition of fuzziness. We should employ them appropriately according to different contexts.

As far as these functions are concerned, fuzzy language can be applied to many fields. For instance, in our daily communication, fuzzy language ensures the accurate information; it is employed in news to improve flexibility; advertisement uses fuzzy words to save time and effort; political and business issues can avoid some sensitive subjects and make the communication polite by the use of fuzzy words; in literature, fuzzy expressions create an aesthetic atmosphere. Nevertheless, in some advertisements businessmen use fuzziness to cheat customers for promoting sales. Therefore, we should keep both positive and negative functions in mind, and take advantage of its positive functions and distinguish the misleading advertisements.

Therefore, translators should take various Pragmatic Functions of Fuzzy Language into their consideration when they do translation work.

\section{References}

Burns, L.C. (1991). Vagueness: An Introduction into Natural Language and the Sorties Paradox. Dardrecht: Kluwer Academic Publisher.

Challel, J. (1994). Vague Language. Oxford: Oxford University. 
David. Crystal. (1991). A Dictionary of Linguistic and Phonetics. Oxford: Blackwell.

Grice, H. P. (1975). Syntax and Semantics. New York: Academic Press.

Guilbaud. (1997). Mathematics and Approximation. Proceeding of the Third International Conference on Mathematics Education. London: Longman.

He, Ziran. (1985). Fuzzy Modifiers and Verbal Communication. Foreign Language, (5).

Lakeoff, G. (1973). Hedges: A Study in Meaning Criteria and the Logic of Fuzzy Concepts. Chicago Linguistic Society Papers. Chicago: Chicago Linguistic Society.

Miao, Dongsheng. (1987). An Introduction to Fuzziness. Press of Chinese People University.

Prince, E. F. \& Bosk, C. (1980). On Hedging in Physician-Physician Discourse. In Linguistic and the Profession. New Jersey: Ablex.

Shi, Anshi. (1988). Fuzzy Semantics and Scalarity. Language of China, (1).

Wu, Tieping. (1999). Fuzzy Linguistic. Shanghai Foreign Languages Press.

Zadeh, L. A. (1965). Information and Control, Vol. 8. Brooklyn, NY: Polytechnic Press.

Zadeh, L. A. (1976). A Fuzzy-Algorithmic Approach to the Definition of Complex or Imprecise Concepts. International Journal of Man-Machine Studies. New York: Academic Press.

Zhang, Qiao. (1998). Fuzzy Semantics. China Social Science Press.

Zhao, Yuanren. (2002). A Collection of Papers on Languages by Zhao Yuanren. Beijing Business Publishing House. 